\title{
SUR LA DÉCOMPOSITION DES PÉNÉDÉTERMINANTS ET DÉTERMINANTS.
}

\author{
Par Maurice Lecat (Watermael).
}

Adunanza del 9 novembre 1919 .

I. Avec L. H. Rice ') disons qu'un indice des éléments d'une matrice est signant (nous conservons le vocable anglais) ou non-signant ${ }^{2}$ ) suivant qu'on tient compte ou non du nombre des inversions dans sa rangée pour trouver les signes des termes du déterminant. Un déterminant de classe $n$ possède le nombre pair

$$
n-\delta_{3,(-1)^{n-1}} \equiv N
$$

d'indices signants, les déterminants de classe impaire ayant $u n$ indice non-signant ${ }^{3}$ )

I) L. H. RICE, P-way Determinants [American Journal of Mathematics, Vol. XL (1918), pp. 242-262], p. 246 .

${ }^{2}$ ) De même pour une rangée, un rang, une direction, un système de files, un système de tranches.

3) Il y a lieu de faire une très importante remarque, qui s'applique à presque tous les travaux qui ont été publiés sur les déterminants de classe supérieure. On entendait par indice fixe (abréviativement pour: indice de la rangée fixe) l'indice ordonné, c'est-à-dire l'indice dont les valeurs dans les éléments d'un terme sont rangées par ordre de grandeur croissante. En cas de classe impaire, on donnait toujours, comme si c'était nécessaire, à l'indice ordonné le rang non-signant, rang qui est imposé (sauf dans des cas spéciaux de mérogénéité). Or, si ce cumul de fonctions et l'emploi d'un vocable à signification double ne sont pas illicites, puisque le choix du rang ordonnateur est arbitraire, ils peuvent présenter plusieurs inconvénients. Signalons-en un: c'est de devoir compter les inversions dans les $n$ - I rangées signantes; tandis que si l'indice ordonné est signant (ce qui a toujours lieu si la classe du déterminant est paire), comme on n'a pas à s'inquiéter des inversions des rangées non-signantes, ce n'est plus que dans $n-2$ rangées qu'il faut compter les transmutations. - R. F. Scotr, L. Gegenbauter et bien d'autres, qui emploient le vocable à double signification, placent toujours l'indice ordonné au premier óu au dernier rangs, ce qui simplifie souvent l'écriture; mais à tort il ne font jamais l'hypothèse que l'indice non-signant occupe une autre place. C'est là une lacune qui a été néfaste dans le développement de la théorie et qui, surtout, combinée, chez certains auteurs, à la méconnaissance de la notion d'indice non-signant, a engendré de nombreuses erreurs. 
et ceux de classe paire n'en ayant pas. Les permanents n'ont, par définition, que des indices non-signants.

On généralise en considérant un nombre quelconque $\vee$ d'indices signants, pourvu que ce nombre reste pair. Cette restriction est imposée par le théorème de CramerBezout sur les permutations d'éléments à indices. Si v était impair le signe d'un terme pourrait changer quand on y permute des éléments.

Le déterminant ainsi généralisé ${ }^{4}$ ) sera appelé pénédéterminani d'espece v. Ces fonctions comprennent, comme cas spécial où $v=N$, les déterminants (full-sign determinants de RICE). On obtient des permanents si $v$ est nul. La plupart des propriétés des déterminants n'appartiennent pas aux permanents, à cause de l'absence d'indice signant dans ces derniers ${ }^{5}$ ).

D’après la définition, le pénédéterminant ${ }^{6}$ )

$$
\left|\left(\varepsilon_{1}, \ldots, \varepsilon_{y} ; \varepsilon_{y+1}, \ldots, \varepsilon_{n}\right)\right|_{p},
$$

où l'on a surmonté des signes $\cap$ et $U$ respectivement les indices signants et nonsignants, les indices de même signance étant supposés contigus pour faciliter l'écriture, peut s'exprimer ainsi :

$$
\begin{aligned}
& \sum_{\omega=1}^{p} \prod_{\sigma=1}^{\nu}\left( \pm_{\omega_{\sigma}}\right) \prod_{\alpha=1}^{p}\left(\omega_{I}^{(\alpha)}, \ldots, \omega_{n-1}^{(\alpha)}, \alpha\right), \\
& \sum_{\varphi=1}^{p} \prod_{s=1}^{\nu-1}\left( \pm_{\varphi_{s}}\right) \prod_{\alpha=1}^{p}\left(\alpha, \varphi_{1}^{(\alpha)}, \ldots, \varphi_{n-1}^{(\alpha)}\right),
\end{aligned}
$$

en plaçant, pour simplifier la forme, l'indice ordonné $\propto$ aux rangs extrêmes.

4) Cette nouvelle extension de la notion de déterminant est très féconde et marque une importante étape dans l'histoire de leur théorie. Car non seulement la plupart des propriétés subsistent quel que soit le nombre des indices signants, mais bien des exceptions disparaissent, dont l'une des plus remarquables est celle que présente la loi de CAYLEY pour multiplier des déterminants par files. Cfr. M. LECAT, Produit de determinants de classes impaires [L'Intermédiaire des Mathématiciens, Vol. XXVI (I9I9), p. 80 et pp. I40-I43]. - On conçoit aisément, et RIce le met en lumière [loc. cit. ${ }^{\mathrm{I}}$ ), p. 255 ], que, à part une très heureuse simplification des notations, les pénédéterminants ne sont autres fonctions que celles que L. Gegenbauer [Einige Sätze über Determinanten hōberen Ranges, Denkschriften der K. Akademie der Wissenschaften in Wien, math.-naturw. Klasse, Bd. LVII (1890), pp. 735-750, $\$$ II] et nous-même [Leçons sur la theorie des determinants à $n$ dimensions (Gand, Hoste, Igro), p. Iog] avions dénommées déterminants-permanents.

5) Par exemple, il y a des matrices particulières dont le pénédéterminant, indépendant de l'espèce, se confond avec le déterminant, mais diffère du permanent.

$\left.{ }^{6}\right)$ Pour simplifier l'écriture, nous reprèsentons l'élément général par $\left(\varepsilon_{1}, \ldots, \varepsilon_{n}\right)$ et non, comme on le fait d'habitude, par $a_{\varepsilon_{1}, \ldots, \varepsilon_{n}}$. 
On peut aussi réprésenter les pénédéterminants par les produits symboliques.

Si l'espèce est $v$, on doit manifestement faire usage, suivant que l'indice ordonné est signant ou non, de $\vee-I$ ou $\nu$ systèmes d'unités alternées $\lambda$ et de $n-\nu$ ou $n-\vee-I$ systèmes d'unités concurrentes $\mu$. . D'où les expressions suivantes:

$$
\begin{aligned}
& \prod_{\alpha=1}^{p} \sum_{\varphi=1}^{p}\left(\alpha, \varphi_{1}^{(\alpha)}, \ldots, \varphi_{n-1}^{(\alpha)}\right) \prod_{s=1}^{\nu=T} \lambda_{\varphi_{s}^{(s)}}^{(s)} \prod_{l=\nu+1}^{n} \mu_{\varphi_{t}^{(\alpha)}}^{(l)}, \\
& \prod_{\alpha=1}^{p} \sum_{\omega=1}^{p}\left(\omega_{1}^{(\alpha)}, \ldots, \omega_{n-1}^{(\alpha)}, \alpha\right) \prod_{\sigma=1}^{\nu} \lambda_{\omega_{\sigma}}^{(\sigma)} \prod_{t=\nu+1}^{n-1} \mu_{\omega_{\tau}^{(\tau)}}^{(\alpha)} \text {. }
\end{aligned}
$$

2. Étudions la décomposition des pénédéterminants en une somme ou en une somme algébrique de pénédéterminants de classe inférieure.

Adoptons le symbole $\Omega^{x}$ pour représenter $\Omega$ ou $u$ suivant que le nombre $x$ est impair ou pair, tout comme $(-I)^{x}$ vaut $(-I)$ ou $(+I)$ suivant les cas.

On a la formule générale suivante de décomposition ${ }^{7}$ ). Le pénédéterminant de classe $l+m+r+s+$ I et d'espece $m+s+\delta_{(-1),(-1)^{m+s}}$ :

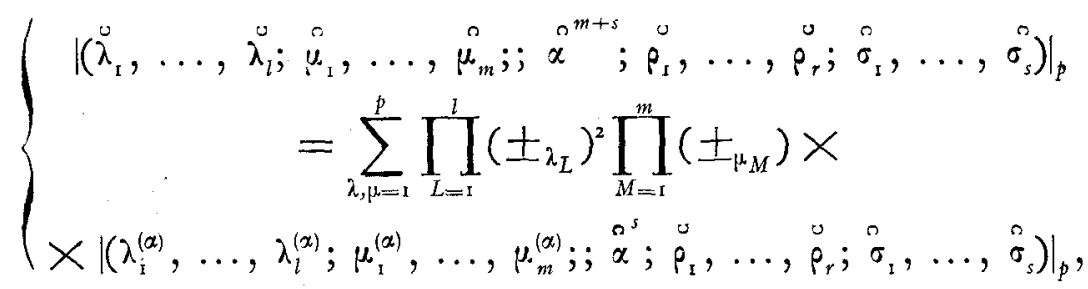

somme algébrique de $(p !)^{l+m}$ composants $\left.{ }^{8}\right)$ de ćlasse $r+s+$ I et d'espèce $s+\delta_{-i,(-1)^{s}}$. Les $l+m$ indices soumis à la sommation ${ }^{9}$ ) prennent tous ${ }^{\text {ro }}$ ) leurs $p$ valeurs dans le système des $p$ tranches correspondant aux $p$ valeurs d'un méme indice $\alpha$ (se trouvant parmi les indices non soumis à la sommation). Le symbole $\left( \pm_{\boldsymbol{\beta}_{M}}\right.$ ) représente ${ }^{\text {II }}$ ) le

7) D'après L. H. Rice, loc. cit. I), p. 249. Nous écrivons la formule d'une manière un peu plus explicite que cet auteur.

8) Components (RICE). Observons que les matrices composantes ne sont autres que celles que nous avons appelées antérieurement espaces transversaux, et qui tous sont des espaces diagonaux lorsque $p=2$.

9) C'est uniquement en vue de faciliter l'écriture et la lecture que l'on a placé consécutivement les indices soumis à la sommation: d'abord les non-signants, puis les signants; ensuite les indices auxquels s'applique le signe sommatoire: l'indice ordonné, puis les indices non-signants, enfin les signants. Mais on conçoit qu'au fond cette disposition ne diminue en rien la généralité.

10) $\mathrm{Au} \mathrm{n}^{\mathrm{O}}$ Io on se libérera de cette importante restriction.

II) Il est à observer que, par convention, le symbole $\left( \pm_{\mu}\right)$ sert non seulement à donner le signe, mais encore à indiquer que les valeurs prises par l'indice $\mu$ doivent être toutes différentés, ce symbole devant être remplacé par zéro si deux ou plusieurs valeurs de $\mu$ étaient égales. En ce qui concerne 
signe de la rangée de l'indice $\mu_{M}$ lorsque la rangée de $\alpha$ est ordonnée ${ }^{\text {12}}$ ) I, $2, \ldots, p$. L'indice $\alpha$ s'appelle la base (terme proposé par RICE).

Mais les signes des composants ne dépendent pas toujours du rang de l'indice $\alpha$. Suivant que le nombre $m$ est pair ou impair, la signance de la base est conservée ou changée, l'espèce devant rester paire après la décomposition ${ }^{\mathbf{1 3}}$ ). Il en résulte que lorsque le nombre des indices signants soumis à la sommation est pair, la base $\alpha$ ne joue plus un rôle spécial utile à mettre en évidence. Dans ce cas il n'y a plus lieu de distinguer cet indice des autres et l'on peut écrire simplement:

$\left|\left(\lambda_{1}, \ldots, \dot{\nu}_{l} ; \hat{\mu}_{1}, \ldots, \dot{\mu}_{m} ; \dot{p}_{1}, \ldots, \dot{p}_{r} ; \dot{\sigma}_{1}, \ldots, \dot{\sigma}_{s}\right)\right|_{p}$
$=\sum_{\lambda, \mu=1}^{p} \prod_{L=1}^{l}\left( \pm_{\lambda_{L}}\right)^{2} \prod_{M=1}^{m}\left( \pm_{\mu_{M}}\right)\left|\left(\lambda_{1}, \ldots, \lambda_{l} ; \mu_{s}, \ldots, \mu_{m} ; \dot{\rho}_{1}, \ldots, \dot{\rho}_{r} ; \dot{\sigma}_{1}, \ldots, \dot{\sigma}_{s}\right)\right|_{p}$,

à condition de sous-entendre (ce qu'il ne faut pas perdre de vue) que les indices soumis à la sommation n'en sont pas moins supposés tous prenfo encore leurs $p$ valeurs dans un même système (arbitraire) de $p$ tranches parallèles.

Il est aisé de voir que, $l, m, r, s$ étant donnés, la décomposition des pénédéterminants peut, dans le cas général de la formule de décomposition $(R)$, se faire d'un nombre de manières donné par l'expression:'

$\Delta \equiv\left[\mathrm{I}+\delta_{-\mathrm{I},(-1)^{m}}\left(r \delta_{-\mathrm{I},(-1)^{s}}+s \delta_{\left.\mathrm{r},(-\mathrm{r})^{s}\right)}\right)\left(\begin{array}{c}l+r+\delta_{\mathrm{I},(-\mathrm{I})^{m+s}} \\ l\end{array}\right)\left(\begin{array}{c}m+s+\delta_{-\mathrm{I},(-1)^{m+s}} \\ m\end{array}\right)\right.$.

3. Considérons des cas particuliers de $(R)$. Si la sommation n'affecte que des indices non-signants, le pénédéterminant est décomposé, non plus en une somme algé-

les indices $l$ non-signants soumis à la sommation (et qui ne donnent pas lieu à des considérations de signes), les $p$ valeurs qu'ils prennent doivent aussi être différentes, ce qu'exprime le symbole $( \pm \lambda)^{2}$. Quand il n'y a aucun inconvénient à sous-entendre ce second symbole, on le supprime pour simplifier l'écriture.

${ }^{2}$ ) La base est ordonnée pour déterminer les signes dont il faut affecter les composants, mais non (il est à peine nécessaire de le faire observer) pour trouver les signes des termes des composants eux-mêmes, opération pour laquelle on peut, en vertu du théorème de CRAMER-Bezout [cfr. la note 3 )], choisir un rang ordonnateur quelconque, signant ou non. Le signe d'un terme n'est pas altéré si l'on permute ses éléments, pourvu que le nombre des indices signants soit pair.

I3) La formule est assez évidente, malgré ou phutôt à cause de sa grande généralité. Cela illustre le fait bien connu (mais sur lequel nous nous plaisons à insister) qu'il est parfois plus aisé de trouver une propriété générale que ses cas particuliers. Ce qui est certain, c'est que l'introduction de la piuricité d'indices non-signants, et par conséquent de la notion de pénédéterminant, a beaucoup facilité la solution de la délicate question du développement des déterminants par abaissement de la classe, c'est-à-dire de la décomposition. 
brique, mais simplement en une "somme » de pénédéterminants. Comme $m$ est alors nul, la signance de l'indice $\approx$ est conservée, et l'on peut écrire simplement que le pénédéterminant de classe $n$ et d'espèce $2 \vee$ :

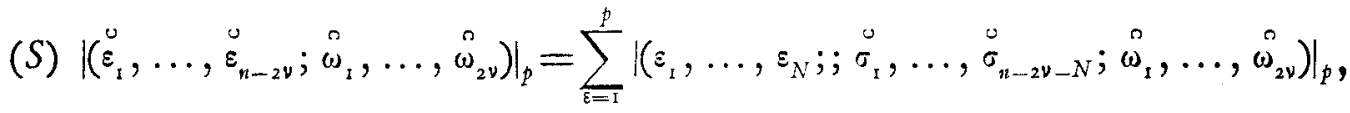
somme de pénédéterminants de classe $n-N$ et d'espèce $2 v$. Et $\Delta=\left(\begin{array}{c}n \\ N\end{array}\right)$.

Si $s$ est nul, le pénédéterminant, d'espèce $m+\delta_{-1,(-1)^{m}}$,

$$
\begin{gathered}
\left|\left(\dot{\lambda}_{1}, \ldots, \dot{\lambda}_{l} ; \hat{\mu}_{1}, \ldots, \hat{\mu}_{m} ; ; \alpha^{n} ; \dot{\rho}_{\mathrm{I}}, \ldots, \dot{\rho}_{r}\right)\right|_{p} \\
=\sum_{\lambda, \mu_{m}=1}^{p} \prod_{M=1}^{m}\left( \pm_{\mu_{M}}\right)\left|\left(\lambda_{r}, \ldots, \lambda_{l} ; \mu_{1}^{(\alpha)}, \ldots, \mu_{m}^{(\alpha)} ; \alpha, \rho_{\mathrm{I}}, \ldots, \rho_{r}\right)\right|_{p},
\end{gathered}
$$

somme algébrique de permanents de classe $r+\mathrm{I}$.

Distinguons deux cas suivant la parité de $m$, autrement dit suivant que tous les indices primitivement signants sont affectés par le signe sommatoire ou bien qu'un de ces indices n'est pas soumis à la sommation. Dans le premier cas on peut écrire simplement que le pénédéterminant de classe $n$ et d'espéce $2 \vee$

$$
\left\{\begin{array}{c}
\left|\left(\omega_{1}^{0}, \ldots, \omega_{2 \nu} ; \varepsilon_{1}, \ldots, \varepsilon_{n-2 \nu}\right)\right|_{p} . \\
\sum_{\omega, \varepsilon=1}^{p} \prod_{\tau=1}^{2 v}\left( \pm_{\omega_{\tau}}\right)\left|\left(\omega_{I}, \ldots, \omega_{2 \nu} ; \varepsilon_{1}, \ldots, \varepsilon_{n-2 v-N} ; ; \varphi_{I}, \ldots, \varphi_{N}\right)\right|_{p}^{\dagger},
\end{array}\right.
$$

somne algébrique de permanents de classe $N$. Et $\Delta=\left(\begin{array}{c}n-2 v \\ n\end{array}\right)$. Si $m$ est impair, la signance de $\alpha$ change et la base n'est plus arbitraire: on est donc conduit à écrire:

$\left(P_{2}\right)\left\{\begin{array}{c}\left|\left(\varepsilon_{1}, \ldots, \stackrel{\varepsilon}{\varepsilon}_{n-2 v-N+1} ; \dot{\omega}_{1}, \ldots, \stackrel{0}{\omega}_{2 v} ; \dot{\varphi}_{1}, \ldots,{\stackrel{\varphi}{\varphi_{N-1}}}_{1}\right)\right|_{p} \\ =\left.\left.\sum_{\varepsilon, \omega=1}^{p} \prod_{\sigma=1}^{2 v-1}\left( \pm_{\omega_{\sigma}}\right)\right|^{\dagger}\left(\varepsilon_{1}, \ldots, \varepsilon_{n-2 v-N+1} ; \omega_{\mathrm{r}}^{(\alpha)}, \ldots, \omega_{2 v-1}^{(\alpha)} ; ; \alpha, \varphi_{1}, \ldots, \varphi_{N_{-1}}\right)\right|_{p},\end{array}\right.$ somme algébrique de permanents de classe $N$. Et l'on voit aisément que $\Delta \equiv 2 v\left(\begin{array}{l}n-2 v \\ N-1\end{array}\right)$. Il y a donc deux formes distinctes pour la décomposition d'un pénédéterminant en permanents.

Si $m+s=o$, l'indice $\alpha$ est de part et d'autre non-signant et la formule signifie que le permanent de classe $n$

$$
\left.{ }_{(i}, \ldots, i_{n}\right)\left.\right|_{p} ^{+}=\sum_{\varepsilon=1}^{p}\left|\left(\varepsilon_{\mathrm{s}}, \ldots, \varepsilon_{n-\nu} ; \omega_{1}, \ldots, \omega_{\nu}\right)\right|_{p}^{+} .
$$

somme de $(p !)^{n-v}$ permanents de classe $v$. 
4. Dans ces divers cas particuliers, les composants sont des pénédéterminants ou des permanents. Voyons maintenant le cas spécial de la décomposition des pénédéterminants suivant des déterminants.

Si $s$ est impair et si $r$ est nul, tous les indices non-signants étant soumis à la sommation, on trouve que le pénédéterminant

$$
\begin{gathered}
\left|\left(\dot{\lambda}_{1}, \ldots, \dot{\lambda}_{l} ; \hat{\mu}_{1}, \ldots, \hat{\mu}_{m} ; ; \hat{\jmath}^{m+1} ; \hat{\sigma}_{1}, \ldots, \hat{\sigma}_{s}\right)\right|_{p} \\
=\sum_{\lambda, \mu=1}^{p} \prod_{M=1}^{m}\left( \pm_{\mu_{M}}\right)\left|\left(\lambda_{1}, \ldots, \lambda_{l} ; \mu_{1}^{(\alpha)}, \ldots, \mu_{m}^{(\alpha)} ; ; \dot{\alpha}, \hat{\sigma}_{1}, \ldots, \hat{\sigma}_{s}\right)\right|_{p},
\end{gathered}
$$

somme algébrique de déterminants de classe paire. Et l'on voit que

$$
\Delta=\left(\mathrm{I}+l \delta_{\mathrm{t},(-\mathrm{r})^{m+\mathrm{r}}}\right)\left(\begin{array}{c}
m+s+\delta_{\mathrm{t},(-1)^{m}} \\
m
\end{array}\right) .
$$

Les composants sont des déterminants à un indice non-signant:

a) si, s étant impair, $r=\mathrm{I}$; ce qui donne:

$$
\begin{gathered}
\left|\left(\dot{\lambda}_{1}, \ldots, \dot{\jmath}_{l} ; \hat{\mu}_{1}, \ldots, \hat{\mu}_{m} ; ; \dot{\alpha}^{m+1} ; \dot{\rho} ; \dot{\sigma}_{\mathrm{r}}, \ldots, \dot{\sigma}_{s}\right)\right|_{p} \\
=\sum_{\lambda, \mu=1}^{p} \prod_{M=1}^{m}\left( \pm_{\mu_{M}}\right)\left|\left(\lambda_{1}, \ldots, \lambda_{l} ; \mu_{1}^{(\alpha)}, \ldots, \mu_{m}^{(\alpha)} ; \dot{\rho} ; \dot{\alpha}, \hat{\sigma}_{1}, \ldots, \hat{\sigma}_{s}\right)\right|_{p}
\end{gathered}
$$

et

$$
\Delta=\left(\mathrm{I}+\delta_{-\mathrm{r},(-1)^{m}}\right)\left(\begin{array}{c}
l+\mathrm{I}+\delta_{1,(-1)^{m+1}} \\
l
\end{array}\right)\left(\begin{array}{c}
m+s+\delta_{1,(-1)^{m}} \\
m
\end{array}\right)
$$

b) si, s étant pair, $r=o$, ce qui fournit:

$$
\begin{gathered}
\left|\left(\dot{\lambda}_{s}, \ldots, \dot{\lambda}_{l} ; \hat{\mu}_{1}, \ldots, \hat{\mu}_{m} ; \dot{\alpha}^{m}, \hat{\sigma}_{1}, \ldots, \hat{\sigma}_{s}\right)\right|_{p} \\
=\sum_{\lambda, \mu=1}^{p} \prod_{M=1}^{m}\left( \pm_{\mu_{M}}\right)\left|\left(\lambda_{1}, \ldots, \lambda_{l} ; \mu_{1}^{(\alpha)}, \ldots, \mu_{m}^{(\alpha)} ; \dot{\alpha}, \hat{\sigma}_{s}, \ldots, \hat{\sigma}_{s}\right)\right|_{p}
\end{gathered}
$$

et

$$
\Delta=\left(\mathrm{I}+s_{-\mathrm{I},(-\mathrm{I})^{m}}\right)\left(\begin{array}{c}
l+\delta_{\mathrm{I},(-\mathrm{I})^{m}} \\
l
\end{array}\right)\left(\begin{array}{c}
m+s+\delta_{\mathrm{I},(-1)^{m+1}} \\
m
\end{array}\right) .
$$

Dans ces trois cas, si $m=0$, on a, au lieu de sommes algébriques, des "sommes » et $\Delta$ se réduit alors respectivement à : I, $l+\mathrm{I}, l+\mathrm{I}$.

5. Envisageons maintenant le cas spécial de la décomposition des déterminants. Servons-nous, en premier lieu, des formules particulières déduites de $(R)$. Voyons d'abord ce que devient la décomposition en une "somme" ( $m$ étant nul). En faisant $v=\mathrm{r}$ dans $(S)$, on voit que le déterminant de classe impaire $2 n+\mathrm{I}$

$$
\left|\left(\varepsilon ; \omega_{\mathrm{I}}, \ldots, \omega_{2 n}\right)\right|_{p}=\sum_{\varepsilon=1}^{p}\left|\left(\varepsilon ; \omega_{1}, \ldots, \omega_{2 n}\right)\right|_{p},
$$


somme (simple) de $p$ ! déterminants de classe paire $2 n$. Cette décomposition n'est possible que d'une seule manière. On voit de plus que ce n'est que si le déterminant primitif a un indice non-signant, que la décomposition peut avoir lieu suivant une "somme".

Si $s$ est nul, $\left(P_{2}\right)$ seule est applicable et on trouve que le déterminant de classe impaire

$$
\left|\left(\omega_{1}, \ldots, \omega_{2 n} ; \varepsilon\right)\right|_{p}=\sum_{\omega=1}^{p} \prod_{\nu=1}^{2 n-1}\left( \pm_{\omega_{\nu}}\right)+\left.\left(\omega_{1}^{(\alpha)}, \ldots, \omega_{2 n-1}^{(\alpha)} ; \alpha, \varepsilon\right)\right|_{p} ^{\dagger},
$$

somme algebrique de permanents ordinaires. Et $\Delta=2 n$. La classe des permanents composants ne peut être supérieure à 2 que pour les pénédéterminants.

6. Étudions, d'après $(R)$, le cas, plus compliqué, où $m s \neq 0$. C'est celui de la décomposition des déterminants en une somme algébrique de déterminants de classe quelconque.

Les classes seront paires si $l=0, r=0, m$ étant pair et $s$ impair; et l'on a simplement :

$$
\left|\left(i_{1}, \ldots, i_{n}\right)\right|_{p}=\sum_{\omega=1}^{p} \sum_{\varepsilon=1}^{2(n-\nu)}\left( \pm_{\omega_{\varepsilon}}\right)\left|\left(\omega_{1}, \ldots, \omega_{2(n-\nu)} ; \sigma_{1}, \ldots, \sigma_{2 \nu}\right)\right|_{p},
$$

décomposition qui est possible de $\left(\begin{array}{l}2 n \\ 2 v\end{array}\right)$ manières.

On obtient la décomposition d'un déterminant de classe paire en déterminants d un indice non-signant, en faisant encore $l+r=0$, mais $m$ étant pris impair et $s$ pair, ce qui donne:

et

$$
\left.\left|\left(i_{1}, \ldots, i_{2 n}\right)\right|_{p}=\sum_{\mu=1}^{p} \prod_{N=1}^{2 n-2 \nu-1}\left( \pm_{\mu_{N}}\right) \mid \mu_{1}^{(\alpha)}, \ldots, \mu_{2 n-2 \nu-1}^{(\alpha)} ; ; \dot{\alpha} ; \sigma_{1}, \ldots, \sigma_{2 \nu}\right)\left.\right|_{p}
$$

$$
\Delta=(2 n+\mathrm{I})\left(\begin{array}{c}
2 n \\
2 v+\mathrm{I}
\end{array}\right)
$$

Pour la décomposition d'un déterminant de classe impaire, quelle que soit la parité de la classe des composants, il y a deux modes suivant que la sommation affecte ou non l'indice primitivement non-signant.

Partons d'abord d'une classe paire. L'indice non-signant est soumis au signe sommatoire si $r=0, l=\mathrm{I}, s$ étant impair et $m$ pair; et l'on a simplement:

$$
\left|\left(\dot{\varepsilon} ; \omega_{1}, \ldots, \omega_{2 n}\right)\right|_{p}=\sum_{\varepsilon, \omega=1}^{p} \prod_{\theta=1}^{2 n-2 \nu}\left( \pm_{\omega_{\theta}}\right)\left|\left(\varepsilon ; \omega_{1}, \ldots, \omega_{2 n-2 \nu} ; ; \sigma_{I}, \ldots, \sigma_{2 \nu}\right)\right|_{p},
$$

décomposition possible de $\left(\begin{array}{l}2 n \\ 2 \vee\end{array}\right)$ manières. Si $n=v$, on retrouve la relation (I) du 
$n^{\circ}$. Le second mode a lieu si l'on fait $r+l=0$ et si l'on prend $m s$ impair. Il faut alors mettre la base en évidence, comme suit:

$$
\left|\left(\omega_{1}, \ldots, \omega_{2 n} ; \dot{\varepsilon}\right)\right|_{p}=\sum_{\omega=1}^{p} \prod_{\tau=1}^{2 n+1}{ }^{-2 \nu}\left( \pm_{\omega_{\tau}}\right)\left|\left(\omega_{\mathrm{r}}^{(\alpha)}, \ldots, \omega_{2 n+1-2 \nu}^{(\alpha)} ; \sigma_{1}, \ldots, \sigma_{2 \nu-1}, \alpha\right)\right|_{p}
$$

et

$$
\Delta=\left(\begin{array}{c}
2 n \\
2 v-1
\end{array}\right)
$$

Voyons enfin la décomposition des déterminants de classe impaire suivant des composants à un indice non-signant. En faisant, dans $(R), l=\mathrm{I}, r=0$, et en prenant $s$ pair et $m$ impair, ce qui exige que l'indice $\alpha$ primitif soit non-signant, on est conduit à écrire:

$$
\left|\left(\dot{\varepsilon} ; \omega_{1}, \ldots, \omega_{2 n}\right)\right|_{p}=\sum_{\varepsilon, \omega=1}^{p} \prod_{\theta=1}^{2 n-2 \nu-1}\left(I_{\omega_{\theta}}\right)\left|\left(\varepsilon ; \omega_{1}^{(\alpha)}, \ldots, \omega_{2 n-2 \nu-1}^{(\alpha)} ; ; \dot{\alpha}, \sigma_{1}, \ldots, \sigma_{2 \nu}\right)\right|_{p},
$$

et l'on a :

$$
\Delta=(2 v+\mathrm{I})\left(\begin{array}{c}
2 n \\
2 v+\mathrm{r}
\end{array}\right)
$$

Le mode où là sommation n'affecte plus l'indice primitivement non-signant s'obtient: soit en faisant $l=0, r=\mathrm{I}$ et en prenant $s$ impair et $m$ pair, $\alpha$ étant primitivement signant; soit en faisant $l+r=0$ et en prenant $s$ et $m$ pairs, $\alpha$ étant primitivement non-signant. Ces deux manières d'opérer, qui ne sont pas listinctes, donnent simplement :

$$
\left|\left(\omega_{1}, \ldots, \omega_{2 n} ; \dot{\varepsilon}\right)\right|_{p}=\sum_{\omega=1}^{p} \prod_{\tau=1}^{2 n-2 \nu}\left( \pm_{\omega_{\sigma}}\right)\left|\left(\omega_{1}, \ldots, \omega_{2 n-2 \nu} ; ; \sigma_{1}, \ldots, \sigma_{2 \nu} ; \dot{\varepsilon}\right)\right|_{p}
$$

et l'on à

$$
\Delta=\left(\begin{array}{l}
2 n \\
2 \vee
\end{array}\right) .
$$

7. EN RÉsumÉ: $\mathrm{I}^{\mathrm{O}}$ Les déterminants à un indice non-signant ont deux modes de décomposition qui n'ont pas lieu si la classe est paire, savoir: a) en une somme de déterminants de classe inférieure d'une unité; b) en une somme algébrique de permanents de classe 2. Dans ce second mode on doit indiquer la base;

$2^{\circ}$ Quelle que soit leur classe, les déterminants peuvent s'exprimer par une somme algébrique de déterminants de classe paire ou impaire quelconque. Il y a un ou deux modes suivant que la classe primitive est paire ou impaire. La base ne doit être mise en évidence: dans le premier cas, que si les composants sont de classe impaire; dans le second cas, que si lindice primitivement non-signant est ou n'est pas soumis à la sommation suivant que les composants sont de classe impaire ou paire.

On peut remarquer, en outre, que quand la base ne doit pas être mise en évi- 
dence, le nombre de manières de décomposer est toujours $\left(\begin{array}{l}2 n \\ 2 \vee\end{array}\right)$, les classes étant $2 n$ ou $2 n+$ I et $2 v$ ou $2 v+$ I $^{14}$ ).

8. En particulier, un déterminant cubique

$$
\left|\left(\varepsilon_{1}, \varepsilon_{2}, \varepsilon_{3}\right)\right|_{p}
$$

peut se décomposer: en une somme de déterminants ordinaires

$$
\sum_{\varepsilon=1}^{p}\left( \pm_{\varepsilon}\right)^{2}\left|\left(\varepsilon ; \omega_{1}, \omega_{2}\right)\right|_{p}
$$

14) Quelques mots d'historique. Tous les auteurs qui se sont occupés de la décomposition des déterminants de classe quelconque, ont traité cette question d'une manière défectueuse, soit qu'ils n'aient considéré que des cas spéciaux et qu'ils aient laissé des lacunes, soit qu'ils aient commis de vraies erreurs. Dans le premier travail (1843) qui ait été publié sur les déterminants à $n$ indices, A. CAYLEY, On the notations and properties of certain fonctions resolvable into a series of determinants [Transactions of the Cambridge Philosophical Society, Vol. VIII (1842-I849), pp. 8;-88]; The Collected Mathematical Papers Vol. I (Cambridge, University Press, 1889), pp. $75-79$ écrit cette égalité (nous conservons littéralement les notations, que le lecteur interprètera aisément sans autre explication):

$$
\left\{\begin{array}{l}
A_{\rho_{1}}, \sigma_{1}, \ldots, \theta_{1}, \varphi_{1}, \ldots,(n) \\
\vdots \\
\vdots \\
\rho_{k}, \sigma_{k}, \ldots, \theta_{k}, \varphi_{k}, \ldots
\end{array}\right\}=\sum \pm_{u} \pm_{\nu} \ldots\left\{\begin{array}{l}
A_{\rho_{I}}, \sigma_{1}, \ldots, \theta_{u_{\mathrm{I}}}, \varphi_{\varphi_{\mathrm{I}}}, \ldots,(n) \\
\vdots \\
\vdots \\
\rho_{k}, \sigma_{k}, \ldots, \theta_{u_{k}}, \varphi_{\varphi_{k}}, \ldots
\end{array}\right\} .
$$

Les auteurs postérieurs, notamment L. Gegenbauer dans son premier Mémoire sur les déterminants de classe supérieure, Ueber Determinanten böheren Ranges [Denkschriften der K. Akademie der Wissenschaften in Wien, math.-naturw. Klasse, Bd. XLIII, Abt. II (1882), pp. I7-32], ne donnent que de vagues indications et ne formulent pas analytiquement la décomposition. Plus tard, L. Gegenbauer, Einige mathematische Theoreme [Sitzungsberichte Akademie Wissenschaften Wien, II ${ }^{\mathrm{a}}$ Abt., Bd. CII (1893), pp. 549-564], p. 562, $\$ 5$, revenant incidemment sur la question; écrit une relation qui, à part la forme (que nous adaptons aux notations du présent travail), n'est autre que

$$
\left|\left(i_{1}, \ldots, i_{n}\right)\right|_{p}=\sum_{\omega=1}^{p} \prod_{\varepsilon=1}^{n-\nu}\left( \pm_{\omega_{\varepsilon}}\right)\left|\left(\alpha, \omega_{1}^{(\alpha)}, \ldots, \omega_{n \rightarrow \nu}^{(\alpha)} ; \sigma_{1}, \ldots, \sigma_{y}\right)\right|_{p},
$$

relation qui, d'après ce qu'on a vu, est exacte moyennant certaines restrictions, mais n'est pas susceptible de contenir tous les cas de décomposition. Gegenbauer s'en sert, comme si elle était. générale, pour donner une nouvelle démonstration, "aussi simple que possible", de la loi de Cayley [loc. cit. ${ }^{4}$ )] sur la multiplication des déterminants par files, renouvelant ainsi une erreur qu'il avait déjà commise plusieurs fois antérieurement, et qui consiste à ne pas observer que la dite loi est inapplicable lorsque les classes des déterminants à multiplier sont toutes deux impaires. Cfr. M. LECAT, loc. cit. ${ }^{4}$ ). La décomposition fut traitée d'une manière encore incomplète dans notre Abrégé de la théorie des déterminants d̀ $n$ dimensions (Gand, Hoste, 19II), pp. 28-30; il n'y est donné qu'un seul mode de décomposition pour les déterminants de classe impaire en composants de classe paire ou impaire, et la décomposition des déterminants de classe paire en déterminants à un indice non-signant n'est pas abordée. 
en une somme algébrique de déterminants ordinaires (ce qui est possible de deux manières)

$$
\sum_{\omega=1}^{p}\left( \pm_{\omega}\right)\left|\left(\alpha, \varepsilon, \omega^{(\alpha)}\right)\right|_{p}, \quad \sum_{\omega=1}^{p}\left( \pm_{\omega}\right)\left|\left(\alpha, \omega^{(\alpha)}, \varepsilon\right)\right|_{p}
$$

ou en une somme algébrique de permanents ordinaires (ce qui est encore possible de deux façons):

$$
\left.\sum_{\omega=1}^{p}\left( \pm_{\omega}\right) \dot{\mid}\left(\varepsilon, \alpha, \omega^{(\alpha)}\right)\right|_{p} ^{+},\left.\left.\quad \sum_{\omega=1}^{p}\left( \pm_{\omega}\right)^{+}\right|^{+}\left(\varepsilon, \omega^{(\alpha)}, \alpha\right)\right|_{p} ^{+} .
$$

Si donc la sommation s'applique à l'indice non-signant, les composants ne contenant pas de normales (c'est-à-dire de files non-signantes), on a une somme de déterminants; si elle affecte un des deux indices signants, on a une somme algébrique de déterminants ou de permanents, suivant que la base est ou n'est pas l'indice primitivement non-signant; autrement dit, suivant que les composants ne contiennent pas de normales ou en contiennent ${ }^{\mathbf{5}}$ ).

Par exemple, le déterminant cubique d'ordre 2 :

$$
\begin{aligned}
& \left|\begin{array}{ll|ll}
\text { III } & \text { IIII } & \text { 2II } & 22 \text { I } \\
\text { II } 2 & \text { I22 } & \text { 2I2 } & 222
\end{array}\right|=\left|\begin{array}{ll}
\text { 1II } & 12 \text { II } \\
2 \text { I2 } & 222
\end{array}\right|+\left|\begin{array}{ll}
2 \text { II } & 22 I \\
1 I 2 & 122
\end{array}\right|
\end{aligned}
$$

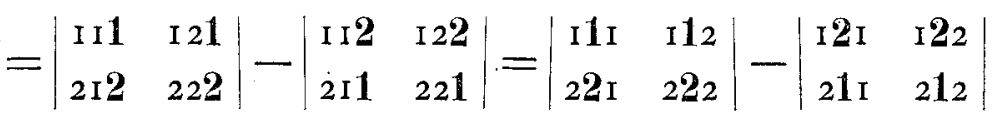

$$
\begin{aligned}
& =\left|\begin{array}{cc}
\mathrm{I} 1 \mathrm{I} & 21 \mathrm{I} \\
\mathrm{I} 22 & 222
\end{array}\right|-\left|\begin{array}{ll}
\mathrm{I} 2 \mathrm{I} & 22 \mathrm{I} \\
\mathrm{I} 12 & 212
\end{array}\right|=\left|\begin{array}{cc}
\mathrm{II} 1 & 2 \mathrm{I} 1 \\
\mathrm{I} 22 & 222
\end{array}\right|-\left|\begin{array}{cc}
\mathrm{II} 2 & 2 \mathrm{I} 2 \\
\mathrm{I} 21 & 221
\end{array}\right| .
\end{aligned}
$$

9. Voyons deux applications de la décomposition des pénédéterminants. Démontrons la propriété suivante:

Un pénédéterminant, de classe quelconque $n$ et d'espèce 2 ,

$$
\left|\left(\varepsilon_{1}^{0}, \ldots, \dot{\varepsilon}_{n-2} ; \stackrel{0}{\omega}_{1}, \stackrel{0}{\omega}_{2}\right)\right|_{p}
$$

à eléments réels, est positif si chaque élément de l'esbace axial à $n-1$ dimensions

$$
\left\|\varepsilon_{1}, \ldots, \varepsilon_{n-2} ; \omega, \omega\right\|_{p}
$$

est positif et supérieur à la somme des valeurs absolues des autres éléments situés sur la même flle d'une direction signante arbitrairement cboisie; c'est-à-dire si, par exemple, la condition:

$$
2\left(\varepsilon_{1}, \ldots, \varepsilon_{n-2} ; \omega, \omega\right)>\sum_{\rho=1}^{p} v \cdot a \cdot\left(\varepsilon_{1}, \ldots, \varepsilon_{n-2} ; \rho, \omega\right)
$$

est remplie pour toutes les valeurs de $\varepsilon$ et de $\omega$. 
En effet, le pénédéterminant peut être décomposé en une somme $(n-2)^{\mathrm{uple}}$

$$
\sum_{\varepsilon=1}^{p}\left|\left(\varepsilon_{1}, \ldots, \varepsilon_{n-2} ; \omega_{1}, \omega_{2}\right)\right|_{p}
$$

de $(p !)^{n-2}$ déterminants ordinaires à vertèbres positives et supérieures chacune à la somme des valeurs absolues des autres éléments de la même file d'une même direction. Or, en vertu d'un théorème de L. LÉvy ${ }^{10}$ ), ces déterminants sont tous positifs. D'où l'on conclut la proposition énoncée.

On voit de même que si les éléments de l'espace axial considéré sont tous négatifs et si la valeur absolue de chacun a la même propriété que précédemment, le pénédéterminant a le signe de $(-1)^{p}$.

S'il n'y a qu'un seul indice non-signant, on a affaire à un déterminant cubique, l'espace axial devenant le plan axial critique ${ }^{17}$ ).

Il est impossible de généraliser le théorème au cas de pénédéterminants à plus de deux indices signants et par conséquent au ćas de déterminants de classe supérieure à 3. Car si la sommation affectait des indices signants, la décomposition se ferait suivant une somme algébrique et la démonstration ne serait plus applicable. Or, dans ces conditions, si les composants n'ont que deux indices signants, le pénédéterminant intégral ne peut en avoir davantage.

Voici une autre application de la décomposition des pénédéterminants. Il résulte de la formule $(S)\left[\begin{array}{ll}\mathrm{n}^{\circ} & 3\end{array}\right]$ que le pénédéterminant de classe $n+N+2 v$ et d'espéce $2 v$ :

$$
\left|\left(\omega_{1}, \ldots, \omega_{2 \nu} ; \Sigma_{1}, \ldots, \varepsilon_{N}\right) \sum_{\alpha=1}^{p}\right|_{\tau=1}^{n}\left|\delta_{\alpha_{\tau}, \theta_{\tau}}\right|_{p}
$$

dont les couches, parallèles de classe $2 \nu+N$, des coordonnées $\omega$ et $\varepsilon$, sont toutes identiques, est égal à

$$
(p !)^{n}\left|\left(\hat{\omega}_{1}, \ldots, \hat{\omega}_{2 \nu} ; \stackrel{0}{\varepsilon}_{1}, \ldots, \stackrel{0}{\varepsilon}_{N}\right)\right|_{p}
$$

15) Les composants sont donc, comme le montre E. R. Hedrick, On Three Dimensional Determinants [Annals of Mathematics, (2), Vol. I (I899-1900), pp. 49-57], pp. 5I-52, des permanents ou des déterminants suivant qu'on décompose par normales ou par files signantes. C'est ce que E. WAELSCH Ueber eine geometrische Behandlungsweise der Elemente der Determinantentheorie [Monatshefte für Ma. thematik und Physik, Bd. IX (1898), pp. 207-214], p. 214, avait exprimé antérieurement, mais avec une tout autre terminologie. Cfr. L. H. Rice, loc. cit. '), pp. 243-244.

16) Ce théorème, qui repose sur la théorie des équations linéaires homogènes, peut s'enoncer ainsi : Un déterminant de classe 2, à élément réels, a le signe de son terme axial, si chaque vertèbre est, en valeur absolue, supérieure à la somme des valeurs absolues des éléments situés sur la même ligne.

17) Nous avons considéré ce cas particulier, dès I9ı. Cfr. Leçons ${ }^{4}$ ), p. 35. 
Cette propriêté généralise celle bien connue des déterminants à strates identiques ${ }^{\mathbf{r} 8}$ ).

ro. Dans tout ce qui précède on a supposé, comme il a été dit au $\mathrm{n}^{\circ} 2$, que tous les indices soumis à la sommation prennent leurs $p$ valeurs dans les $p$ tranches correspondant aux $p$ valeurs de la base. L. H. Rice ${ }^{\text {t9}}$ ) démontre qu'on peut se libérer de cette condition, moyennant une restriction.

Les indices soumis à la sommation sont répartis en groupes d'étendues quelconques, mais en nombre égal à la classe $v$ des composants. On obtient les nouvelles matrices composantes en faisant prendre dans le groupe de rang $s$ les $p$ valeurs de chacun de ses indices, dans les $p$ tranches (à $v-I$ dimensions) de rangs $k_{s}$, en prenant successivement $s=\mathrm{I}, \ldots, v$, et en choisissant pour $k_{\mathrm{r}}, \ldots, k_{v}$ une permutation des nombres $I, \ldots$, v. La restriction est que le nombre des indices signants ne peut être impair que dans un seul groupe. La condition nécessaire et suffisante pour que cette imparité ait lieu dans un groupe est évidemment que le nombre des rangs primitivement signants parmi les indices non soumis au signe sommatoire soit lui-même impair. L'indice correspondant à ce groupe spécial joue alors le rôle essentiel qui était attribué antérieurement à la base $x$ (quand elle devait être mise en évidence).

Ces nouveaux composants, dits croisés (crossed components), ont les mêmes signes que les composants primitifs, et la somme algébrique des composants croisés est encore égale au pénédéterminant (ou au permanent) primitif. On voit qu'il ne peut $y$ avoir décomposition croisée si la classe de la matrice primitive est inférieure à 5 .

En vue de faciliter l'écriture et la lecture, supposons, ce qui ne diminue pas au fond la généralité, que $k_{s}=s$; de plus, écrivons encore consécutivement les indices soumis à la sommation, les indices d'un même groupe étant consécutifs, les indices signants suivant les non-signants. Supposons, en outre, que la base $\alpha_{1}$ soit au premier rang. La décomposition croisée d'un pénédéterminant est la suivante:

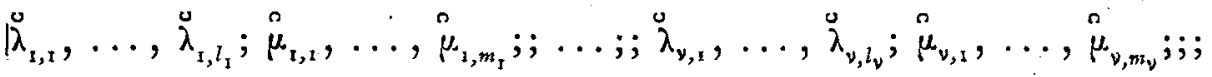

$$
\begin{aligned}
& \left.\hat{\alpha}_{1}^{\nu-N+1} ; \ddot{\alpha}_{2}, \ldots, \dot{\alpha}_{N} ; \hat{\alpha}_{N+1}, \ldots, \hat{\alpha}_{\nu}\right)\left.\right|_{p} \\
& =\sum_{\lambda, \mu=1}^{p} \prod_{\varepsilon=1}^{\nu} \prod_{M_{\varepsilon=1}}^{m_{\varepsilon}}\left( \pm_{\mu_{M_{\varepsilon}}}\right) \mid\left(\lambda_{\mathrm{s}, 1}^{\left(\alpha_{1}\right)}, \ldots, \lambda_{\mathrm{I}, l_{1}}^{\left(\alpha_{1}\right)} ; \mu_{\mathrm{I}, \mathrm{I}}^{\left(\alpha_{1}\right)}, \ldots, \mu_{\mathrm{r}, m_{1}}^{\left(\alpha_{\mathrm{s}}\right)} ; \ldots ;\right.
\end{aligned}
$$

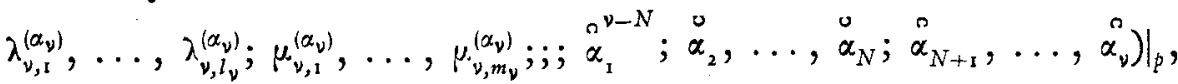

tous les $m$ devant être pairs sauf $m_{1}$.

${ }^{18}$ ) On pourrait de même étendre au cas des pénédéterminants la propriété, plus générale (cfr. nos Leçons 4), pp. 79-81), relative aux déterminants ayant des strates identiques, mais non toutes.

19) L. H. RICE, loc. cit. ${ }^{x}$ ), pp. $252-254$. 
Si $m_{1}$ aussi est pair, il devient inutile de distinguer spécialement l'indice $\alpha_{1}$ et l'expression se simplifie donc. Nous pouvons nous dispenser de l'écrire.

Si $M \equiv \sum_{\varepsilon=1}^{v} m_{\varepsilon}=0$, on a une « somme » de composés croisés.

Si $\vee-N=0$, on obtient la décomposition croisée d'un pénédéterminant (d'un permanent, si en outre $m$ est nul) en une somme algébrique (en une somme) de permanents.

En ce qui concerne la décomposition croisée des pénédéterminants suivant des déterminants, et celle des déterminants eux-mêmes suivant une somme algébrique de permanents de classe 2 ou de déterminants de classe quelconque, ou encore suivant une "somme " de déterminants de classe inférieure d'une unité, elles ne présentent aucune particularité remarquable par rapport à ce qu'on a vu aux $n^{\text {os }} 4,5,6$.

Watermael, le 30 août 1919.

MAURICE LECAT. 